\title{
RF diffraction effect in RF-induced thermoacoustic tomography: calibration and distortion
}

Changhui Li, Geng Ku, Pramanik Manojit, Lihong V. Wang

Changhui Li, Geng Ku, Pramanik Manojit, Lihong V. Wang, "RF diffraction effect in RF-induced thermoacoustic tomography: calibration and distortion," Proc. SPIE 6856, Photons Plus Ultrasound: Imaging and Sensing 2008: The Ninth Conference on Biomedical Thermoacoustics, Optoacoustics, and Acousto-optics, 685612 (28 February 2008); doi: 10.1117/12.763450 


\title{
RF Diffraction Effect in RF-induced Thermoacoustic Tomography: Calibration and Distortion
}

\author{
Changhui Li, Geng Ku, Pramanik Manojit and Lihong V. Wang \\ Optical Imaging Lab, Department of Biomedical Engineering \\ Washington University in St. Louis, St. Louis, MO, 63130
}

\begin{abstract}
Because the wavelengths of radio-frequency (RF) waves used in thermoacoustic tomography (TAT) are comparable with the size of detected objects, RF diffraction plays important roles in TAT. The RF diffraction affects not only the global distribution of the RF field in the tissue, but also local RF energy deposition. In this paper, we discussed these two major effects. Both numerical simulations and phantom experiments are done to demonstrate these phenomena. We also provide a partial correction method for the image distortion and a calibration algorithm for image calibration.
\end{abstract}

Keywords: RF diffraction, thermoacoustic tomography, image distortion, image calibration

\section{INTRODUCTION}

Due to the thermal expansion effects after the tissue absorbs the radiation energy of a RF pulse, tissue generates acoustic signals. Thermoacoustic (TA) tomography (TAT) reconstructs the TA source distribution from the detected acoustic signals over the tissue surface. TAT uses radio frequency electromagnetic (EM) waves as the illumination source, and it combines the sensitive RF contrast and high ultrasound resolution. TAT has the advantage over photoacoustic tomography (PAT) that it can image deeper into tissue because the RF attenuation coefficient of soft tissue is much smaller than the optical counterpart. TAT has become an important and promising imaging modality, especially for early breast cancer detections. TAT has been studied for both laboratory research and clinical applications in breast cancer imaging. ${ }^{1-4}$

The acoustic pressure $p$ excited by a RF pulse can be described by ${ }^{5,6}$

$$
\left(\nabla^{2}-\frac{1}{c^{2}} \frac{\partial^{2}}{\partial t^{2}}\right) p(\mathbf{r}, t)=-\frac{\beta}{C_{p}} \frac{\partial H(\mathbf{r}, t)}{\partial t},
$$

where $H(\mathbf{r}, t)$ is a heating function defined as the thermal energy deposited at spatial position $\mathbf{r}$ and time $t$ by the electromagnetic (EM) radiation per unit time per unit volume, $\beta$ is the isobaric volume expansion coefficient in $K^{-1}, C_{p}$ is the specific heat in $J /(K k g)$ and $c$ is the acoustic speed. In this paper, $\beta, C_{p}$ and $c$ are assumed to be uniform constants.

The EM energy absorbed by tissue per unit time per unit volume is calculated by

$$
H(\mathbf{r}, t)=\sigma(\mathbf{r})|\mathbf{E}(\mathbf{r}, t)|^{2},
$$

where $\sigma$ is the conductivity, $\mathbf{E}(\mathbf{r}, t)$ is the real electrical field denoted by a vector

$$
\mathbf{E}(\mathbf{r}, t)=\left(\begin{array}{c}
E_{x}(\mathbf{r}, t) \\
E_{y}(\mathbf{r}, t) \\
E_{z}(\mathbf{r}, t)
\end{array}\right),
$$

"|..." represents the magnitude of a vector. Since $\sigma$ in tissue is generally dispersive, equation (2) is exact only for monochromatic source illumination. Because the RF pulse has a finite bandwidth, we must consider the dispersive effect.

Further author information: (Send correspondence to Changhui Li) Changhui Li: E-mail: cli@biomed.wustl.edu, Telephone: 13149359586

Photons Plus Ultrasound: Imaging and Sensing 2008: The Ninth Conference on Biomedical Thermoacoustics, Optoacoustics, and Acousto-optics, edited by Alexander A. Oraevsky, Lihong V. Wang, Proc. of SPIE Vol. 6856, 685612, (2008) · 1605-7422/08/\$18 · doi: 10.1117/12.763450 


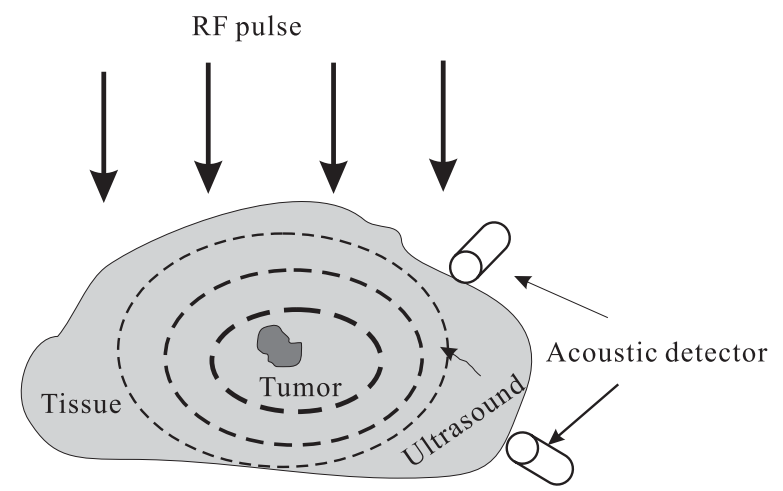

Figure 1. Illustration of thermoacoustic tomography. A pulsed RF wave generated from a radar penetrates the tissue from top. Due to the dielectric contrast, malignant tissue (such as a tumor) usually absorbs more RF energy and produces a higher temperature increase than the surrounding normal tissue. Then thermoelastic expansion leads to the generation of ultrasound. Acoustic detectors receive the ultrasound signals around the tissue, which are used to reconstruct the RF absorption distribution.

From electrodynamics, the rate of the energy absorption from the EM field per unit volume and per unit time in the tissue can be calculated by the dot product of the current density and electric field. Assuming all absorbed energy generates heat, we can write the generalized formulation of the heating function as

$$
H(\mathbf{r}, t)=\mathbf{j}(\mathbf{r}, t) \cdot \mathbf{E}(\mathbf{r}, t) .
$$

Here $\mathbf{j}$ is the real current density, which is related to the conductivity as

$$
\mathbf{j}(\mathbf{r}, t)=\int_{-\infty}^{+\infty} \hat{\sigma}(\mathbf{r}, \omega) \tilde{\mathbf{E}}(\mathbf{r}, \omega) e^{-i \omega t} d \omega,
$$

where $\omega$ is the angular frequency of the field and a variable with an overhead symbol denotes its counterpart in the frequency domain. The "hat" over the conductivity means $\hat{\sigma}$ generally represents a $3 \times 3$ real matrix, which describes the property of an anisotropic medium. To simplify the problem, we assume that the tissue is isotropic. Then, the conductivity becomes a real scalar. Therefore, Eq. (4) can be rewritten as

$$
\mathbf{j}(\mathbf{r}, t)=\int_{-\infty}^{+\infty} \sigma(\mathbf{r}, \omega) \tilde{\mathbf{E}}(\mathbf{r}, \omega) e^{-i \omega t} d \omega
$$

In our experimental system, the RF source transmits 3.0-GHz center-frequency and 0.5- $\mu$ s wide pulses. Since the temporal width of the pulse is 1000 times longer than the EM field oscillation period at the center frequency, the source has a narrow bandwidth, and so does the internal field E. We further assume that the conductivity varies slowly within the bandwidth. Thus, we have the following approximation:

$$
\sigma(\mathbf{r}, \omega) \approx \sigma\left(\mathbf{r}, \omega_{c}\right)
$$

where $\omega_{c}=2 \pi f_{c}$ and $f_{c}=3.0 \mathrm{GHz}$. Therefore, the current density can be approximately written as

$$
\begin{aligned}
\mathbf{j}(\mathbf{r}, t) & \approx \sigma\left(\mathbf{r}, \omega_{c}\right) \int_{-\infty}^{+\infty} \tilde{\mathbf{E}}(\mathbf{r}, \omega) e^{-i \omega t} d \omega \\
& =\sigma\left(\mathbf{r}, \omega_{c}\right) \mathbf{E}(\mathbf{r}, t) .
\end{aligned}
$$

Substituting Eq. (6) into Eq. (3), we obtain

$$
\begin{aligned}
H(\mathbf{r}, t) & \approx \sigma\left(\mathbf{r}, \omega_{c}\right) \mathbf{E}(\mathbf{r}, t) \cdot \mathbf{E}(\mathbf{r}, t) \\
& =\sigma\left(\mathbf{r}, \omega_{c}\right)|\mathbf{E}(\mathbf{r}, t)|^{2} .
\end{aligned}
$$


Due to the limitation in the bandwidth of the acoustic transducer, the Giga hertz EM frequency is far out of the transducer's response region, the detected signal corresponds to a short time average of the heating function. From our previous work in, ${ }^{19}$ the short-time average of the local heating function with the narrow bandwidth approximation is

$$
<H(\mathbf{r}, t)>\approx \sigma\left(\mathbf{r}, \omega_{c}\right) \gamma\left(\mathbf{r}, \omega_{c}\right) I_{e}(t),
$$

where $\gamma\left(\mathbf{r}, \omega_{c}\right)$ is the distribution factor, and $I_{e}$ is the RF illumination intensity.

TAT reconstructs images according to the heating function $\langle H(\mathbf{r}, t)\rangle$. Previous studies show that large differences exist in conductivity and permittivity between excised malignant and normal breast tissues. ${ }^{7-11}$ Thus $\sigma\left(\mathbf{r}, \omega_{c}\right)$ contains information about the position and the shape of a malignant breast tissue object. In previous literature, it was assumed that the reconstructed images represent the real structure of the sample. From Eq. (8), this assumption is valid only if $\gamma\left(\mathbf{r}, \omega_{c}\right) I_{e}(t)$ is uniform inside the target (such as a tumor). However, this assumption is generally untrue due to the RF diffraction. On the other hand, the illumination field $I_{e}$, which the local object feels, varies in space too, so does the corresponding magnitudes of the TAT images. Thus the TAT images need to be calibrated in order to do quantitative study.

In this paper, firstly, we give a brief description of the simulation method used in our research. Then we discussed the image distortion and image calibration for TAT separately, in which both numerical simulations and phantom experiments were provided. Finally, there is a conclusion.

\section{SIMULATION METHOD}

The RF sources used in TAT have frequencies ranging from several hundreds $\mathrm{MHz}$ to several GHz. The corresponding wavelengths are from meters to centimeters, which are comparable to the size of the interested objects (such as an adult breast), and thus the diffraction effects governed the EM transportation. Since EM diffraction is beyond geometric optics, we use the finite-difference time-domain (FDTD) ${ }^{13,14}$ technique to perform the numerical simulations. The FDTD method, originally developed by $\mathrm{Yee}^{13}$ in 1966, is suitable for simulating radiative interactions with particles of complex morphology in the diffraction region. The basic principle of this method is to compute the discretized version of the two Maxwell's curl equations:

$$
\begin{aligned}
& \nabla \times \mathbf{H}=\frac{\partial \mathbf{D}}{\partial t}+\vec{j} \\
& \nabla \times \mathbf{E}=-\frac{\partial \mathbf{B}}{\partial t},
\end{aligned}
$$

where $\mathbf{E}, \mathbf{D}$ are the electric field and displacement, respectively; $\mathbf{H}, \mathbf{B}$ are the magnetic field and magnetic flux, respectively; $\vec{j}$ represents the electrical current density, which equals to $\sigma \mathbf{E}$ in the tissue. The electric and magnetic fields are spatially discretized. Then the second order leapfrog algorithm is used to discretize both the spatial and temporal derivatives in Eq. (9). As an example, Eq. (10) gives the time-domain iteration formula for $\mathbf{E}_{\mathbf{x}}$ and $\mathbf{H}_{\mathbf{x}}$ fields in the vacuum $\left(\vec{j}=0, \mathbf{D}=\epsilon_{0} \mathbf{E}\right.$ and $\mathbf{B}=\mu_{0} \mathbf{H} . \epsilon_{0}$ and $\mu_{0}$ are the vacuum's permittivity and permeability, respectively). To simplify the case, we choose same spatial intervals along $\hat{x}, \hat{y}$ and $\hat{z}$ directions: $\Delta x=\Delta y=\Delta z=\Delta s$.

$$
\begin{aligned}
& E_{x}^{n+1}\left(I, J+\frac{1}{2}, K+\frac{1}{2}\right) \\
= & E_{x}^{n}\left(I, J+\frac{1}{2}, K+\frac{1}{2}\right)+\frac{\Delta t}{\epsilon_{0} \Delta s} \\
\times & \left\{H_{z}^{n+1 / 2}\left(I, J+1, K+\frac{1}{2}\right)-H_{z}^{n+1 / 2}\left(I, J, K+\frac{1}{2}\right)\right. \\
+ & \left.H_{z}^{n+1 / 2}\left(I, J+\frac{1}{2}, K\right)-H_{z}^{n+1 / 2}\left(I, J+\frac{1}{2}, K+1\right)\right\} \\
& H_{x}^{n+1 / 2}\left(I+\frac{1}{2}, J, K\right) \\
= & H_{x}^{n-1 / 2}\left(I+\frac{1}{2}, J, K\right)+\frac{\Delta t}{\mu_{0} \Delta s}
\end{aligned}
$$




$$
\begin{aligned}
& \times\left\{E_{z}^{n}\left(I+\frac{1}{2}, J-\frac{1}{2}, K\right)-E_{z}^{n}\left(I+\frac{1}{2}, J+\frac{1}{2}, K\right)\right. \\
& \left.+\quad E_{y}^{n}\left(I+\frac{1}{2}, J, K+\frac{1}{2}\right)-E_{y}^{n}\left(I+\frac{1}{2}, J, K-\frac{1}{2}\right)\right\},
\end{aligned}
$$

where $\Delta t$ is the temporal interval, and the superscripts of the fields represent the discretized time-domain indices.

In practice, the computational domain is bounded by proper boundary condition layers. The EM fields within the domain are simulated by the finite-difference analog of Maxwell's equations. The presence of a dielectric object in the computational domain is specified by properly assigning permittivity and conductivity values at grid points. In this manner, the FDTD method is flexible in simulating various radiative interactions involving complex object morphologies and system configurations.

We used the the uniaxial perfect matched layer (UPML) ${ }^{15}$ boundary condition to absorb outgoing EM waves in our code. Its accuracy is shown in $^{16}$ by comparing the simulated results of light scattering in vacuum with the analytical results based on the Mie theory. Since tissue, such as breast fat, is also RF absorbing, we modified the UPML boundary based on the method in ${ }^{17}$ to match the absorbing medium.

\section{IMAGE DISTORTION}

\subsection{Numerical Simulations For Image Distortion}

The phantoms used in the simulation a homogenous dielectric cylinders of radii $4.5 \mathrm{~mm}$ and height of $20 \mathrm{~mm}$. The permittivity and conductivity of the phantom are

$$
\epsilon_{r}=55, \sigma=2.0 \mathrm{~S} / \mathrm{m} .
$$

The phantom is assumed to be immersed in the mineral oil, which has a permittivity of 2.2 and almost zero conductivity. The phantom is illuminated by a $\hat{x}$-polarized RF plane wave, with frequency of $3.0 \mathrm{GHz}$, from the bottom as shown in Fig. 2(a).

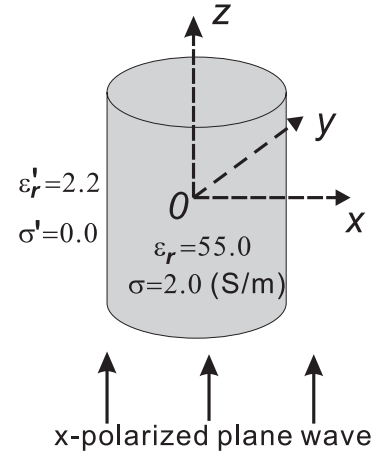

(a)

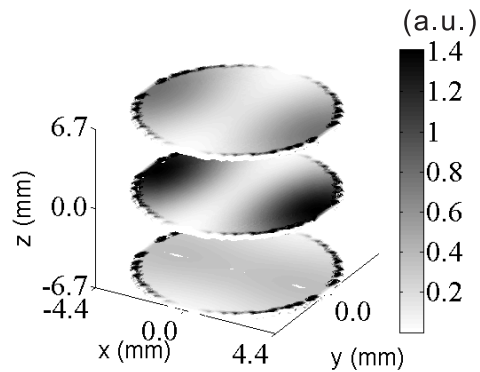

(b)

Figure 2. Simulation model and simulated result. (a) The cylinder has a radius of $4.5 \mathrm{~mm}$ and height of $20 \mathrm{~mm}$. The relative permittivity of the phantom is 55 and the conductivity is $2.0(\mathrm{~S} / \mathrm{m})$. The phantom is immersed in the mineral oil bath, which has the relative permittivity of 2.2 and zero conductivity. The RF illumination is a plane wave with $\hat{x}$ polarization. (b) The simulated energy absorption pattern at three horizontal cross sections.

Figure 2(b) shows the 3D simulation result of the energy deposition within the phantom. We plotted the simulation result on three horizontal cross section at $z=0.0, \pm 6.7(\mathrm{~mm})$, respectively. It is obviously to see that the energy absorption pattern inside the phantom is nonuniform, and a clear "split" pattern along $\hat{x}$ direction shows up.

The simulation result in Fig. 2(b) demonstrates a possible image distortion: "image splitting" along the local polarization direction (it may not be the same as the polarization direction of the original illumination source). Thus, the image of a homogenous object could look like an aggregation of two (or more) different objects. 
Distortion patterns in TAT images are not limited to this "split" pattern. As discussed in,${ }^{19}$ image distortion patterns change with the shape, orientation, surrounding medium and illumination polarization. The presence of image distortion in TAT brings new challenges to interpret the results.

\subsection{Experiment for Image Distortion}

Experiments were carried out to demonstrate the image distortion in TAT. The experiment setup is shown in Fig. 3. Phantoms are immersed in mineral oil, which has low RF absorption and provides good acoustic coupling. A transducer scans around the phantom during the experiment. Figure 4(a) shows two cylindrical phantoms made

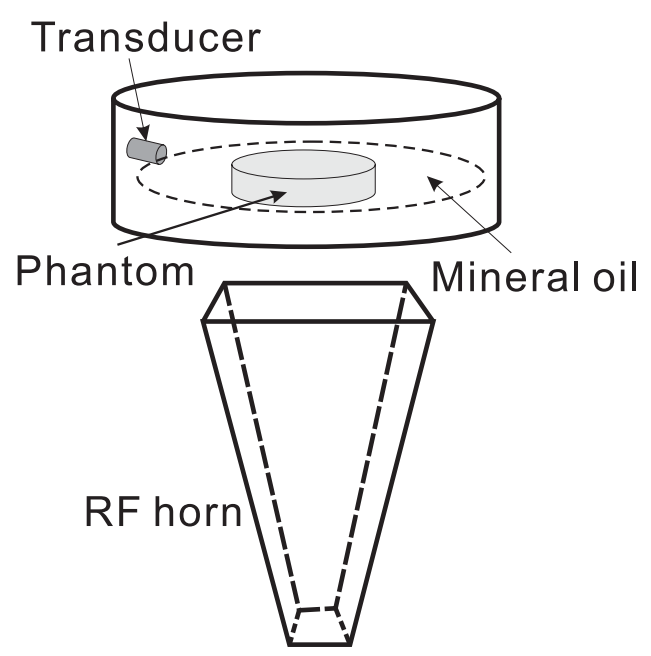

Figure 3. Experiment setup. The phantom and transducer are placed in a tank filled with the mineral oil. Microwave is illuminating upward through the horn. The dashed circle represents the scanning trajectory.

of agar gelatin. One has a radius of $4.5 \mathrm{~mm}$ and another $3.0 \mathrm{~mm}$. They all have the same depth of $20 \mathrm{~mm}$. Both samples are made of $2 \%$ agar gelatin and $98 \%$ water. There is a $2.0-\mathrm{cm}$ separation between two phantoms.

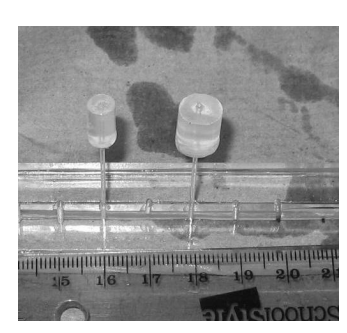

(a)

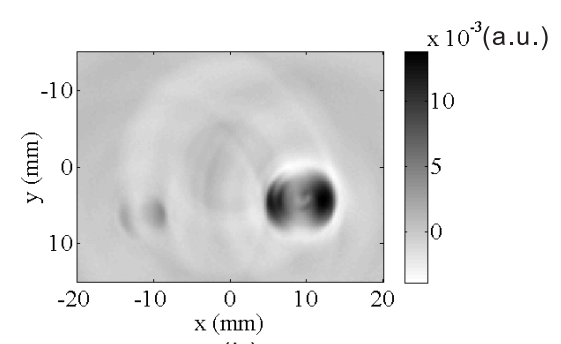

(b)

Figure 4. Two agar gel phantoms and the reconstructed image. (a) Two phantoms made of $2 \%$ agar and $98 \%$ water. One has a radius of $4.5 \mathrm{~mm}$ and another $3.0 \mathrm{~mm}$. They have the same length of $20 \mathrm{~mm}$. Both phantoms are fixed straightly upward by fibers during the experiment. The illumination source has a $\hat{x}$ polarization. (b) The reconstructed image.

A 3.0-GHz 0.5- $\mu s$ RF pulse source was used in the experiment. The source included a commercial magnetron (Marconi, MG5223) and home-made circuit. It had a repetition rate of $100 \mathrm{~Hz}$ to irradiate the sample using an air-filled pyramidal horn type antenna (WR284 horn antenna W/EEV flange, HNL Inc.) with an opening of $7.34 \times 10.69 \mathrm{~cm}^{2}$ and $T E_{10}$ mode. The pulse energy was estimated to be around $10 \mathrm{~mJ}$. For detecting the ultrasound signal, we used a $2.25 \mathrm{-MHz}$ central frequency, $1.27-\mathrm{cm}$ diameter active area unfocused transducer (ISS $2.25 \times 0.50 \mathrm{NF}$, Krautkramer). The transducer was scanned around the sample on a plane perpendicular to the gel cylinder axis for a full 360 degrees, with 160 stops. At each stop, the signal was first amplified by a low-noise pulse amplifier (5072PR, OlympusNDT), then filtered electronically and finally recorded using an oscilloscope (TDS640A, Tektronix). The signal was recorded 50 times at each stop, then averaged to reduce 
noise and transferred to a PC for image reconstruction. A function generator (10MHz pulse generator 4010, Global Specialties) triggered the RF pulses and synchronized the sampling of the oscilloscope.

A simple delay-and-sum reconstruction algorithm ${ }^{18}$ was used to reconstruct the $2 \mathrm{D}$ images from the acquired data (shown in Fig. 4(b)). Because the thickness of the image slice is $\sim 12.7 \mathrm{~mm}$ (about the diameter of the transducer's active area), the images correspond to the overlap of the patterns of different layers in Fig. 2.

Significant distortions are shown in Fig. 4(b) when the image is compared to the actual phantom sample (Fig. 4(a)). The image of the phantom with radius of $4.5 \mathrm{~mm}$ is in good consistent with previous simulation result as shown in Fig. 2(b). Although both agar phantoms located symmetrically in experiment, so they are almost equally illuminated, and both reconstructed images have the "split" pattern, they have huge differences in image magnitudes. The image of the smaller one have much less values than the larger one. This means the signal strengths coming from objects with different shapes, but with same dielectric properties, could be very different. Thus, not only the image could be distorted, the image magnitude depends on the shape and size too.

The distortion patterns could be very diverse from case to case. Fig. 5 presents another example. The phantom used here is a porcine fat with rectangular and triangular cross section cuttings, in which the same agar gelatin as in 4(a) are filled. Fig. 5(b) shows a heavily distorted image. From the distorted image, it is hardly to achieve the phantom original shape.

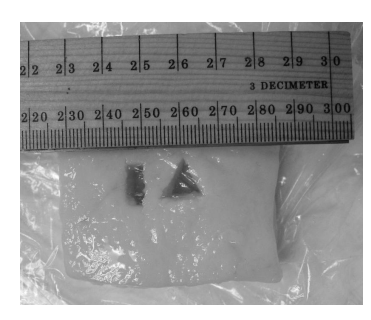

(a)

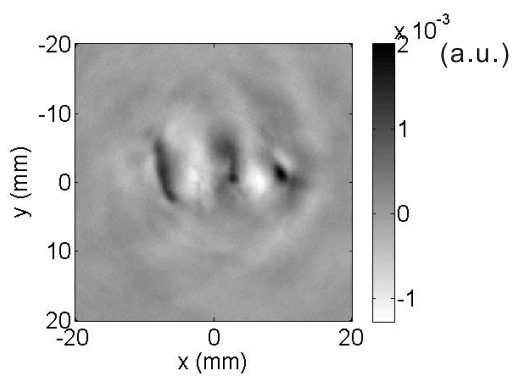

(b)

Figure 5. Another phantom and its reconstructed image. (a) The phantom is a porcine fat with two cuttings, one has a rectangular cross section and another triangular shape. (b) The reconstructed image with polarization along $\hat{x}$.

We already provided a partial correction method to alleviate the image distortion in TAT in. ${ }^{19}$ In that method, two images are required to obtain separately by two perpendicular illumination polarizations. Then a third image can be achieved by averaging these two images. It is equally to get a TAT image by using a circular polarized RF source. This partial correction method helps the correction for "split" pattern, but it is not valid with certainty as discussed in ${ }^{19}$ for relative larger objects.

\section{IMAGE CALIBRATION}

\subsection{Numerical Simulation for Image Calibration}

The TA signal proportionally depends on the illumination strength. Because the RF diffraction affects the global distribution of the RF illumination fields, signals generated by the same kind of TA sources but at different locations could be very different. Thus the TAT images need to be calibrated in order for quantitatively anaclasis.

Field distributions can be obtained through numerical simulation. The simulation must consider not only the phantom itself, but also the source, experiment setup and environmental conditions. All these factors play important roles in determining the field distribution. Based on our experiment setup in Fig. 3, as an example, we simulated the field distribution with a porcine fat phantom of $10 \mathrm{~cm}$ in diameter and $1.5 \mathrm{~cm}$ in depth.

From the numerical simulation, a 3D "ap" of illumination field distribution inside the phantom can be obtained. We call this map "calibration map". The calibration map will be further used to calibrate the reconstructed TAT images from the raw data. 


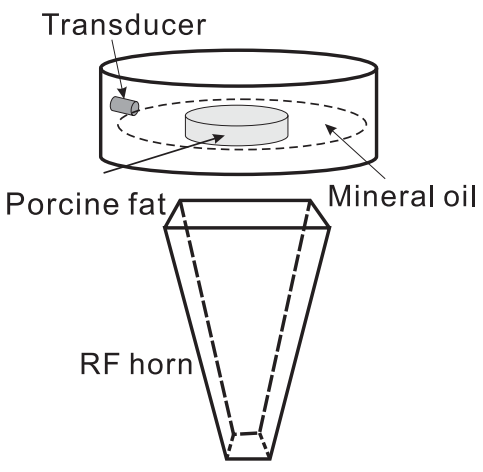

(a)

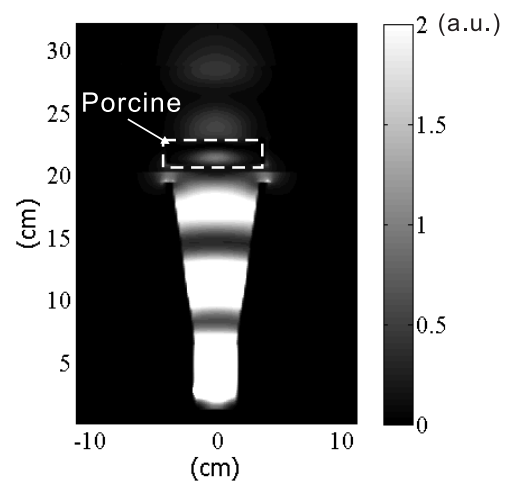

(b)

Figure 6. The experiment setup and the RF field distribution. (a) The experiment setup as in Fig. 3. (b) The RF field intensity within the whole system. The white dashed rectangle encloses the region of a porcine fat phantom.

\subsection{Calibration Algorithm}

A complete calibration algorithm is

1. Find the dielectric properties of the normal breast tissue;

2. Determine the simulation boundary conditions;

3. Run numerical simulations to obtain the calibration map.

4. Modify the reconstructed images based on the field distribution.

Once we obtained the calibration map as describe in the above subsection, the TAT images can be calibrated by dividing the reconstructed image by the calibration map.

\subsection{An Example for Image Calibration}

The phantom is a $1.5 \mathrm{~cm}$ thick and about $10 \mathrm{~cm}$ in diameter porcine fat, on which there are five holes with same diameters of $6 \mathrm{~mm}$. All holes are filled with gel made of $2 \%$ agar and $98 \%$ water. From the simulation, we

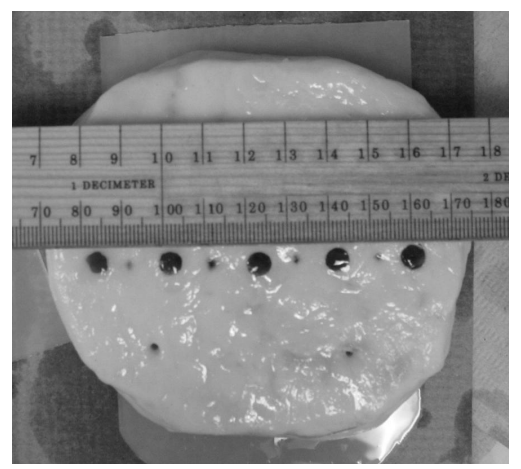

Figure 7. The phantom is a porcine fat with $1.5 \mathrm{~cm}$ in thickness and $10 \mathrm{~cm}$ in diameter. Five holes are drilled at the same diameter of $6 \mathrm{~mm}$. Holes are filled with agar gel with $2 \%$ agar and $98 \%$ water.

obtained the calibration map, Fig. 8(a) shows a 2D cross section of the map. The calibration map clearly shows the nonuniform distribution of the illumination field inside the phantom. Fig. 8(b) shows the field intensity changes along the center line $(y=0)$ of the porcine fat phantom. The field intensity changes very small within the region less $2 \mathrm{~cm}$ away from the center, and quickly decreases outside that region. Thus we expected the TAT images shows similar pattern. 


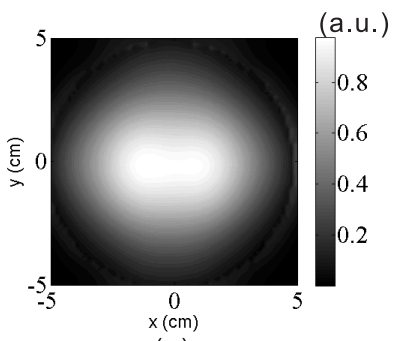

(a)

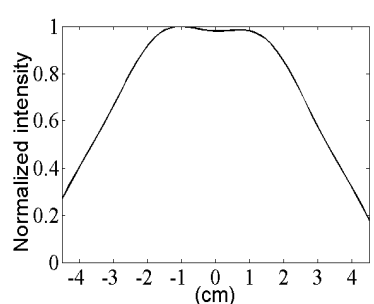

(b)

Figure 8 . The phantom is a porcine fat with $2 \mathrm{~cm}$ in thickness and $10 \mathrm{~cm}$ in diameter. Five holes are drilled at the same diameter of $6 \mathrm{~mm}$. Holes are filled with agar gel with $2 \%$ agar and $98 \%$ water.

Fig. 9(a) is the reconstructed image before the calibration. The splitting pattern is due to the image distortion mechanism discussed in previous section. As we expected, those middle three images, which are within $2 \mathrm{~cm}$ from the center, have similar magnitudes. The rest two images at two ends have much less magnitudes in value. To get correctly quantitative dielectric information of the phantom, images in Fig. 9(a) must be calibrated.

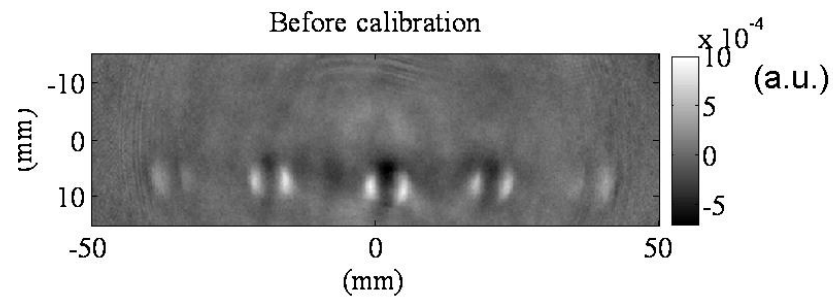

(a)

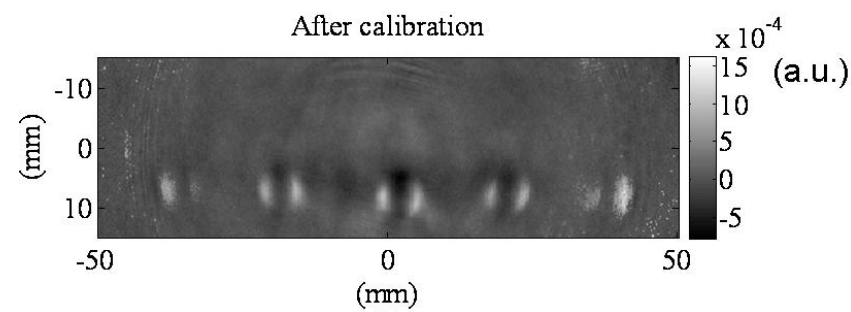

(b)

Figure 9. Images before and after the calibration. (a) TAT image reconstructed from the raw data. (b) The calibrated image obtained by dividing 9 (a) by the calibration map in Fig. 8(a).

To calibrate the TAT image, we divided Fig. 9(a) by Fig. 8(a), then we obtained Fig. 9(b). After calibration, it is clearly that the magnitudes of the two weak images were substantially increased, and the other images keep unchanged.

\section{CONCLUSIONS AND DISCUSSION}

In this paper, we studied the RF diffraction effects in thermoacoustic tomography. Our theoretical analysis and phantom experiment results show that TAT images not only need to be calibrated, but also could be distorted. The image distortion depends not only on the dielectric properties of the object itself, it also depends on the shape, orientation of the object, as well as the dielectric properties of the surrounding medium. We provided a partial correction method to alleviate the image distortion, however, a complete correction method needs more future studies. We also provided and demonstrate a calibration algorithm.

Although we used a monochromatic source approximation in this paper, the discussions in the introduction can be straightforwardly generalized to study wide band illuminations. 


\section{REFERENCES}

1. R. A. Kruger, K. Kopecky, A. Aisen, D. Reinecke, G. Kruger, and W. K. Jr., "Thermoacoustic ct with radio waves: A medical imaging paradigm," Radiology 221, pp. 275-278, 1999.

2. R. Kruger, W. K. Jr., D. Reinecke, G. Kruger, and R. Eisenhart, "Thermoacoustic computed tomography of the breast at $434 \mathrm{mhz}, "$ IEEE MTT-S Int. Microwave Symp. Dig. 2, pp. 591-594, 1999.

3. L. Wang, X. Zho, H. Sun, and G. Ku, "Microwave-induced acoustic imaging of biological tissues," Review of Scientific Instruments 70, pp. 3744-3748, 1999.

4. G. Ku and L. V. Wang, "Scanning thermoacoustic tomography in biological tissue," Med. Phys. 27, pp. 11951202,2000 .

5. V. E. Gusev and A. A. Karabutov, Laser Optoacoustics, AIP, New York, 1993.

6. G. J. Diebold, T. Sun, and M. I. Khan, "Photoacoustic monopole radiation in one, two, and three dimensions," Phys. Rev. Lett 67, p. 3384, 1991.

7. S. Chaudhary, R. Mishra, A. Swarup, and J. Thomas, "Dielectric properties of normal and malignant human breast tissues at radiowave and microwave frequencies," Indian J. Biochem.Biophys. 21, pp. 76-79, 1984.

8. A. J. Surowiec, S. S. Stuchly, J. R. Barr, and A. Swarup, "Dielectric-properties of breast-carcinoma and the surrounding tissues," IEEE Transactions on Biomedical Engineering 35(4), pp. 257-263, 1988.

9. W. T. Joines, Y. Zhang, C. X. Li, and R. L. Jirtle, "The measured electrical-properties of normal and malignant human tissues from 50 to 900 mhz," Medical Physics 21(4), pp. 547-550, 1994.

10. M. Lazebnik, D. Popovic, L. McCartney, C. B. Watkins, M. J. Lindstrom, J. Harter, S. Sewall, T. Ogilvie, A. Magliocco, T. M. Breslin, W. Temple, D. Mew, J. H. Booske, M. Okoniewski, and S. C. Hagness, "A large-scale study of the ultrawideband microwave dielectric properties of normal, benign and malignant breast tissues obtained from cancer surgeries," Physics in Medicine and Biology 52(20), pp. 6093-6115, 2007.

11. M. Lazebnik, L. McCartney, D. Popovic, C. B. Watkins, M. J. Lindstrom, J. Harter, S. Sewall, A. Magliocco, J. H. Booske, M. Okoniewski, and S. C. Hagness, "A large-scale study of the ultrawideband microwave dielectric properties of normal breast tissue obtained from reduction surgeries," Physics in Medicine and Biology 52(10), pp. 2637-2656, 2007.

12. S. Semenov, R. Svenson, A. Boulyshev, A. Souvorov, V. Borisov, Y. Sizov, A. Starostin, K. Dezern, G. Tatsis, and V. Baranov, "Microwave tomography: two-dimensional system for biological imaging," IEEE Trans. Bio-Med. Eng. 43, pp. 869-877, 1996.

13. K. S. Yee, "Numerical solution of initial boundary value problems involving maxwell's equations in isotropic media," IEEE Trans. Antennas Propagat. AP-14, pp. 302-307, 1966.

14. A. Taflove and S. Hagness, Computational Electrodynamics: the Finite-Difference Time-Domain Method, Artech, Boston, MA, 2000.

15. Z. S. Sacks, D. M. Kingsland, R. Lee, and J. F. Lee, "A perfect matched anisotropic absorber for use as an absorbing boundary condition," IEEE Trans. Antennas Propag. 43, pp. 1460-1463, 1995.

16. C. Li, G. W. Kattawar, and P. Yang, "Effects of surface roughness on light scattering by small particles," Quant. Spectrosc. Radiat. Transfer 89, pp. 123-131, 2004.

17. W. Sun, N. G. Loeb, and Q. Fu, "Finite-difference time-domain solution of light scattering and absorption by particles in an absorbing medium," App. Opt. 41, pp. 5728-5743, 2002.

18. C. G. A. Hoelen, F. F. M. de Mul, R. Pongers, and A. Dekker, "Three-dimensional photoacoustic imaging of blood vessels in tissue," Opt. Lett. 23(8), pp. 648-650, 1998.

19. C. Li, M. Pramanik, G. Ku, and L. V. Wang, "Image Distortion in Thermoacoustic Tomography Caused by Microwave Diffraction," submitted to Physical Review E, 2007. 